\title{
Ought to the Changes within the Immunophenotype of Solid Cell Nests (SCNs) and Follicular Cells in Hashimoto's Thyroiditis be Considered as Premalignant Lesions?
}

\section{Tran Ngoc Dung}

Military Medical Hospital 103

Nguyen Khac Tuyen

Military Medical Hospital 103

Truong Dinh Tien

Military Medical Hospital 103

Pham Van Thinh

Military Medical Hospital 103

Nhu Binh Do

Military Medical Hospital 103 https://orcid.org/0000-0002-1377-3921

Nguyen Khanh Van

Military Medical Hospital 103

Nguyen Manh Hung

Military Medical Hospital 103

Nguyen Thuy Linh ( $\boldsymbol{D}$ bsnguyenthuylinhc6@gmail.com )

https://orcid.org/0000-0003-4326-1991

Research article

Keywords: Solid cell nests, Hashimoto's Thyroiditis, Papillary thyroid carcinoma

Posted Date: May 19th, 2020

DOl: https://doi.org/10.21203/rs.3.rs-28265/v1

License: (c) (1) This work is licensed under a Creative Commons Attribution 4.0 International License. Read Full License 


\section{Abstract}

Background: Papillary thyroid carcinoma (PTC) is more frequently reported in patients with Hashimoto's thyroiditis (HT), which may be associated with the presence of solid cell nests (SCNs) and focal PTC-like nuclear alterations in the thyroid gland. The point of this consideration was to assess the morphological and immunohistochemical features of SCNs and follicular epithelial changes in Vietnamese patients with HT.

Materials and methods: Hematoxylin - Eosin and immunohistochemistry were performed on 20 samples of HT patients who underwent thyroidectomy and were diagnosed with Hashimoto's thyroiditis at Military Medical Hospital 103 from 6/2018 to 6/2019. The expression of five markers (P63, Calcitonin, TTF1, CK19, and HBME-1) to be up-regulated in SCNs and follicular epithelial changes were evaluated.

Results: $90 \%$ of samples had SCNs with an average of 10 SCNs per section. Only type 1 and type 4 of SCNs were presented ( $85 \%$ and $55 \%$, respectively) and all SCNs were composed of main cells (p63positive). 15 of 18 cases having SCNs possessed nuclear features of PTC. C-cell hyperplasia was found in one case with 20 clusters. All SCNs showed strong staining with CK19 and weak staining with HBME-1. Follicular epithelial changes were Hürthle cell metaplasia, PTC-like nuclear alterations, atypical solid nodules, papillary and glomerular-like forms $(40 \%, 100 \%, 25 \%$, and $50 \%$, respectively). Follicular cells of glomerular-like forms (new alteration) especially were positive with CK19 $(2+\sim 3+)$, HBME-1 (1+), and TTF1, while the components in these follicles were negative with CK19, HBME-1, and TTF1. Among PTClike nuclear alterations, all of the atypical solid nodules related to HT showed markers related to PTC and without SCNs.

Conclusions: Increasing the number of SCNs, as well as PTC-like nuclear alterations of main cells in SCNs and follicular epithelial changes, which were co-expressed CK19 and HBME-1, may suggest precancerous changes in Hashimoto's thyroiditis.

\section{Introduction}

Hashimoto's thyroiditis (HT) at first portrayed by Dr. Hakaru Hashimoto in 1912, is an autoimmune syndrome of the thyroid gland. The main histological feature of $\mathrm{HT}$ is the infiltration of primary lymphocytes, organized in lymphoid follicles that frequently appear noticeable germinal centers. Other highlights incorporate Hürthle cell change, destruction and atrophy of follicular cells, and interstitial fibrosis [1-4].

It is reported that there was a relationship between Hashimoto's thyroiditis and thyroid cancer. In particular, the relative percentage range in the literature was $0-30 \%$ [5-9]. The increased frequency of cancer (especially PTC) related to HT according to the writing recommends that whether HT is a risk factor of thyroid carcinoma or not [9],[10]. In terms of etiology, PTC is related to HT because both diseases may be derived from the same populace of p63-positive pluripotent stem cells within the title of solid cell nests (SCNs). In 1907, SCNs were first described by Getzowa, who defined that SCNs were small 
thyroid structures and remnants during embryonic development of the human body. SCNs were also considered as small, morphologically inconspicuous aggregates and positive with p63, which were popular in $\mathrm{HT}[11]$.

The follicular epithelial changes that occur in HT have been emphasized since its original description in 1912 [12]. Except for classical histological features as the most important criteria to make the diagnosis, another unusual feature of HT which has been mentioned as a potential source of confusion for diagnosis is focal clear nuclear change with some degree of overlapping comparable to that perceived in PTC [13]. The presence of atypical follicular cell changes in HT has been already said within the writing without an in-depth consideration of their highlights and/or conceivable noteworthiness.

We examined 20 specimens of Hashimoto's thyroiditis. Their blocks were stained by H.E and IHC to assess the characteristics of morphology and immunohistochemistry of the SCNs and the modified follicular cells on the infiltration of the lymphocytic background.

\section{Materials And Methods}

\section{Materials}

All cases accessioned under the diagnosis of Hashimoto's thyroiditis within the surgical pathology files at the Department of Histopathology, Military Medical Hospital 103 from 6/2018 to 6/2019 were reviewed. Of a total of 860 cases on which thyroid surgery was done, HT was found incidentally in 20 patients who underwent lobectomy or subtotal thyroidectomy for other reasons, including thyroid tumors, as well as multinodular goiter. In comparison to H.E staining slides, we selected carefully one sample from more than two samples of each patient, according to the same criteria such as a typical HT on H.E staining slides and a two-dimensional sample size of $1 \times 1 \mathrm{~cm}$. Hematoxylin - Eosin and immunohistochemistry stainings for P63, Calcitonin, TTF1, CK19, and HBME-1 were performed on 20 samples. The expression of five markers to be up-regulated in Hashimoto's thyroiditis.

\section{Methods}

We checked on all the accessible slides of each case to affirm the conclusion of HT, assess its expansion, and characterize of follicular cell changes. The age and sex of all the patients were recorded. As it were cases that appeared the classic pathologic highlights of $\mathrm{HT}$, comprising of atrophic follicles with inexhaustible Hürthle cells but no or diminished colloid and extensive lymphocytic infiltrate with germinal center formation. The degree of the thyroid inclusion by HT was assessed and classified as central or diffuse, as already portrayed within the writing [8]. Cases with as it were lymphocytic penetrated without the other components of HT were avoided from the consideration.

Immunohistochemical staining of the Leica detection framework was performed on successive segments ( $3 \mu \mathrm{m}$ thick) from each documented tissue block. Essential antibodies, sources, and weakenings are 
recorded in Table 1.

Table 1

Five antibodies used in the study

\begin{tabular}{|c|c|c|c|c|c|c|c|}
\hline Anti-body & Species & $\begin{array}{l}\text { Catalog } \\
\mathbf{N}^{0}\end{array}$ & Clone & $\begin{array}{l}\text { Antibody } \\
\text { Concentration }\end{array}$ & Source & Company & Country \\
\hline \multirow[t]{2}{*}{ CK 19} & Mouse & PA0799 & B170 & $0.07 \mathrm{mg} / \mathrm{L}$ & Mono & Leica & UK \\
\hline & $\begin{array}{l}\text { Anti- } \\
\text { human }\end{array}$ & & & & & & \\
\hline \multirow[t]{2}{*}{ HBME-1 } & & PA0458 & HBME1 & $\begin{array}{l}\text { Not } \\
\text { applicable }\end{array}$ & Mono & Leica & UK \\
\hline & $\begin{array}{l}\text { Anti- } \\
\text { human }\end{array}$ & & & & & & \\
\hline \multirow[t]{2}{*}{ TTF1 } & Mouse & PA0364 & SPT24 & $2.6 \mathrm{mg} / \mathrm{L}$ & Mono & Leica & UK \\
\hline & $\begin{array}{l}\text { Anti- } \\
\text { human }\end{array}$ & & & & & & \\
\hline Calcitonin & Mouse & PA0406 & POLYCLONAL & $\begin{array}{l}\text { Not } \\
\text { applicable }\end{array}$ & mono & Leica & UK \\
\hline \multirow[t]{2}{*}{ p63 } & Mouse & PA0103 & 7JUL & $20 \mathrm{mg} / \mathrm{L}$ & Mono & Leica & UK \\
\hline & $\begin{array}{l}\text { Anti- } \\
\text { human }\end{array}$ & & & & & & \\
\hline
\end{tabular}

According to the manufacture's instruction, all sections were deparaffinized with xylene, rehydrated through a series of descending graded alcohols. Antigen retrieval was performed in $1 \mathrm{mmol} / \mathrm{L}$ EDTA PH 8.0. Sections did not get the antigen retrieval program with HBME-1. Endogenous peroxidase activity was blocked by using $3 \% \mathrm{H} 2 \mathrm{O} 2$ for $20 \mathrm{~min}$. They were then incubated with primary antibody for 1 hour at room temperature, followed by a peroxidase-conjugated Polymer (Leica, United Kingdom) for 30 min. To reveal the immunostaining, we had used the sections which were incubated with the Leica REAL DAB + Chromogen for 5 min (Leica, United Kingdom), then counterstained with hematoxylin, followed by dehydration and mounting. Slides were examined using a BX50 optical microscope (Olympus, Japan) with taking photos by iPhone X (Apple Inc, USA).

Appropriate positive controls were used for cytokeratin 19, p63, TTF1, HBME-1, Calcitonin. We evaluated the expression CK19, HBME-1 on IHC staining:

$1(+)$ : the whole membrane color is weak or incomplete.

$2(+)$ : the whole-cell membrane color is average

$3(+)$ : the whole-cell membrane color is strong 
We examed p63 staining in all slides to mark SCNs and compared IHC with H.E specimens to count and identify characterized expression patterns of SCNs. SCNs were classified into 4 types according to Asioli in 2009 [14]. We also assessed the expression of Calcitonin, TTF1, CK19, HBME-1 on SCNs, and PTC-like changes in Hashimoto's thyroiditis.

\section{Ethical considerations}

The Ethical Review Committee of Military Medical University, Vietnam approved the protocol of the study. The study was in line with the Declaration of Helsinki. Written informed consent has been signed by all participants after full explanation.

\section{Statistical analysis}

The collected data were processed by the method of medical statistics using Microsoft Excel 2010 software

\section{Results}

All patients were female, aged from 28 to 69 (53 \pm 12$)$ years old. The age group of $46-60$ years was the most prevalent (40\%).

\section{Morphological characteristics of SCNs}

A summary of the morphological features of SCNs in Table 2. There were a total of 18 slides (90\%) having SCNs. The number of SCNs per section ranging from 0 to 35 (median 10 nests) divided into three groups: The 1st group with zero SCNs per section, the 2nd group 1-3 SCNs per section and the 3rd group with over 3 SCNs per section (10\%, 30\%, and 60\%, respectively). Only have type 1 and type 4 SCNs were seen in our patients ( $85 \%$ and $55 \%$, respectively).

\begin{tabular}{|llll|}
\hline Characteristics & & Number & Ratio \\
\hline Number of SCNs & 0 & 2 & $10 \%$ \\
\cline { 2 - 3 } & $1-3$ & 6 & $30 \%$ \\
\cline { 2 - 3 } & $>3$ & 12 & $60 \%$ \\
\hline Type & 1 & $10 \pm 10.3$ & \\
& 4 & 11 & $55 \%$ \\
\hline PTC-like nuclear changes in SCNs & $15 / 18$ & $83.3 \%$ \\
\hline
\end{tabular}

Table 2

Morphological characteristics of SCNs 
Most SCNs were found near or in the lymphoid follicles and there was one case in which SCNs located between thyroid follicles. The majority of SCNs had changes in the nucleus, including nuclear enlargement, chromatin clearing, and nuclear groove (83.3\%). Some examples of HT with the morphological feature of SCNs are shown in Fig. 2.

\section{Histopathological characteristics of follicular cell changes}

\begin{tabular}{|llll|}
\hline \multirow{2}{*}{ Characteristics } & \multicolumn{2}{l}{ Location of follicular cell changes } & \multirow{2}{*}{ Total } \\
\cline { 2 - 4 } & $\begin{array}{l}\text { Diffuse } \\
(\mathbf{n}=\mathbf{8})\end{array}$ & Surrounded lymphoid follicles $(\mathbf{n = 1 2 )}$ & \\
\hline Hürthle cell metaplasia & $6(30 \%)$ & $2(10 \%)$ & $8(40 \%)$ \\
\hline PTC-like nuclear alteration & $8(40 \%)$ & $12(60 \%)$ & $20(100 \%)$ \\
\hline Papillary structure & $3(15 \%)$ & $2(10 \%)$ & $5(25 \%)$ \\
\hline Glomerular-like structure & $7(35 \%)$ & $3(15 \%)$ & $10(50 \%)$ \\
\hline
\end{tabular}

Table 3

Histopathological characteristics of follicular epithelial changes in Hashimoto's Thyroiditis As shown in Table 3, of all cases with HT, there were 8 (40\%) cases showed diffuse involvement and 12 $(60 \%)$ cases that showed focal involvement of follicular cell changes surrounding lymphoid follicles. When we observed slides on the optical microscope of 20 cases, four changes were reported such as Hürthle cell metaplasia, PTC-like nuclear alterations, papillary structure, glomerular-like structure (Fig. 3). In all cases, PTC-like nuclear alterations accounted for the majority (60\%). Compared with the focal lesson, papillary and glomerular-like forms encountered more frequently in diffuse lesions.

PTC-like nuclear alterations detected in 20 (100\%) were composed of two types of changes, including follicular formed by PTC-like nuclear cells and atypical solid nodules. The papillary structure defined as short papillary with fibrovascular cores was detected in 5 (25\%). $50 \%$ of cases had glomerular-like structures that were grown from thyroid follicles which were damaged by lymphocytic infiltrations and inflammatory cells of interstitial tissues invade into these follicles forming the components in them similar to the glomerulus. Characteristics of glomerular - like structure are enlarged follicles, atrophy of follicular cells which can also be PTC-like nuclear features. The component of glomeruli involves lymphocytes, macrophages, and fibrocytes of interstitial tissue.

\section{The immunohistochemical characteristics in SCNs and follicular cell changes}

The immunohistochemical results for HBME-1, CK19, TTF1, p63, and Calcitonin in the SCNs are summarized in Table 4. 


\begin{tabular}{|c|c|c|c|c|c|}
\hline Marker & \multicolumn{3}{|c|}{ Positive (Number/Ratio) } & Negative (Number/Ratio) & Total \\
\hline P63 & \multicolumn{3}{|l|}{$18(100 \%)$} & 0 & 18 \\
\hline Calcitonin & \multicolumn{3}{|l|}{0} & $18(100 \%)$ & 18 \\
\hline \multirow[t]{2}{*}{ TTF1 } & \multicolumn{3}{|l|}{$18(100 \%)$} & 0 & 18 \\
\hline & $(1+)$ & $(2+)$ & $(3+)$ & & \\
\hline CK19 & 0 & 0 & $18(100 \%)$ & 0 & 18 \\
\hline HBME-1 & $16(88,9 \%)$ & $1(5,6 \%)$ & 0 & $1(5,6 \%)$ & 18 \\
\hline
\end{tabular}

Table 4

Characteristics of the expression of immune markers in SCNs

When analyzing the results of immunohistochemistry with five markers, p63-positive expression in SCNs was detected in 18 cases, while SCNs were negative for Calcitonin in all cases (Fig. 4). In 18 cases which had SCNs, all SCNs were positive with TTF1, local and strongly positive with CK19; local and weakly positive with HBME-1 expression in 16 cases (88.9\%). Co-expression of 2 markers (CK19 and HBME-1) was observed in $16(94.4 \%)$ of 18 cases having SCNs.

The staining intensity of the above five markers in follicular cell changes was summarized in Table 5. All follicular cell changes were negative for p63, Calcitonin, but positive for TTF1. CK19 was frequently stained weakly or moderately in Hürthle cell metaplasia and papillary structures (50\% and $60 \%)$. By contrast, frequencies of strongly CK19-positive staining were higher for PTC-like nuclear alterations and glomerular-like structures (95\% and 30\%). Among these PTC-like nuclear alterations, all atypical solid nodules expressed intensively with CK19. The recoloring intensity of HBME-1 was frequently negative or weakly positive in 50\% (4/8) Hürthle cell metaplasia, 95\% (19/20) PTC-like nuclear alterations, 80\% (4/5) papillary structures and 100\% (10/10) glomerular-like structures, in comparison 50\% (4/8) Hürthle cell metaplasia, 5\% (1/20) PTC-like nuclear alterations, 20\% (1/5) papillary structures were moderate positive with HBME-1. Co-expression of 2 markers (CK19 and HBME-1) was seen in all lessons. 


\begin{tabular}{|c|c|c|c|c|}
\hline Marker & \multicolumn{3}{|c|}{$\begin{array}{l}\text { Positive } \\
\text { (Number/Ratio) }\end{array}$} & $\begin{array}{l}\text { Negative } \\
\text { (Number/Ratio) }\end{array}$ \\
\hline P63 & \multicolumn{3}{|l|}{0} & $20(100 \%)$ \\
\hline Calcitonin & \multicolumn{3}{|l|}{0} & $20(100 \%)$ \\
\hline TTF1 & \multicolumn{3}{|l|}{$20(100 \%)$} & 0 \\
\hline CK19 & $(1+)$ & $(2+)$ & $(3+)$ & 0 \\
\hline Hürthle cell metaplasia (8) & $4(50 \%)$ & $4(50 \%)$ & 0 & 0 \\
\hline PTC-like nuclear alteration (20) & 0 & $1(5 \%)$ & $19(95 \%)$ & 0 \\
\hline Papillary structure (5) & $3(60 \%)$ & $2(40 \%)$ & 0 & 0 \\
\hline Glomerular-like structure (10) & 0 & $5(50 \%)$ & $3(30 \%)$ & $2(20 \%)$ \\
\hline HBME-1 & $(1+)$ & $(2+)$ & $(3+)$ & \\
\hline Hürthle cell metaplasia (8) & $4(50 \%)$ & $4(50 \%)$ & 0 & 0 \\
\hline PTC-like nuclear alteration (20) & $19(95 \%)$ & $1(5 \%)$ & 0 & 0 \\
\hline Papillary structure (5) & $3(60 \%)$ & $1(20 \%)$ & 0 & $1(20 \%)$ \\
\hline Glomerular-like structure (10) & $8(80 \%)$ & 0 & 0 & $2(20 \%)$ \\
\hline
\end{tabular}

Table 5

Characteristics of the expression of five markers in follicular epithelial changes

In the glomerular-like structures, only their follicular cells that were positive with CK19 and HBME-1, TTF1 and the component of glomeruli were negative for CK19, HBME-1, and TTF1. Heterogeneous expression of CK19 was reported frequently in each lesson (Fig. 5).

\section{Discussion}

\section{Morphological and immunohistochemical characteristics of solid cell nests}

SCNs, identified as small nests of cells, are similar to squamous cells or transitional epithelium. Such nests surrounded by stroma usually differ clearly from the thyroid follicles. Multiple foci (2-8 per section) of SCNs are common. [15]. SCNs are found incidentally within the ordinary thyroid gland and may be related to the neoplastic or non-neoplastic injuries of the thyroid gland, the incidence of SCNs in adults is from $3 \%$ of routinely examined thyroids to $60 \%$ of the glands subjected to serial blocking [16]. SCNs have been reported in $9.5-41 \%$ of patients with multinodular thyroid diseases [17]. The association between focal lymphocytic thyroiditis (FLT) and SCNs was reported in 1985 by Prod'hom when the author examined the thyroid tissue of 500 routine autopsies. SCNs were found in $56(11.2 \%)$ of all cases and 18 (30.5\%) of 59 FLT cases [18]. In our study, SCNs were found in $90 \%$ of patients with Hashimoto's 
thyroiditis. This incidence was much higher than that of SCNs in the normal thyroid gland. Our result with an average of $10(0-35)$ SCNs per section was similar to the figure of other authors when finding more than ten SCNs per slide on microscopic examinations [14].

According to Asioli (2009), SCNs are classified into four types. In our study, 20 cases of Hashimoto's thyroiditis had only type 1 and type 4 , accounting for $85 \%$ and $55 \%$. Type 1 (floret-like) is composed of main cells, characterized by round to oval or elongated cells with scant cytoplasm, centrally located oval to fusiform nuclei and occasional nuclear grooves (Fig. 2A). Type 4 SCNs (mixed follicles) have a follicular appearance and contain both follicular cells and small (Fig. 2C). While type 2 was extremely recognized in H.E staining slides and type 3 did not present in any sections.

SCNs are composed of two cell populations - predominant main cells (positive for p63) and a minority of C-cells (positive for Calcitonin). Main cells are defined as polygonal/elongated to round to spindle cells with squamoid features (but no intercellular bridges). Their nuclei are centrally located, oval to fusiform, with finely granular chromatin, uneven nuclear membrane, occasional nuclear grooves. In contrast, C-cells a minor population of cells with clear cytoplasm and centrally located, small compact nuclei. In our study on 20 patients with $\mathrm{HT}$, all of the cells in SCNs were main cells because of p63 expression (Fig. 2B, D) Besides, we found that there was only a patient who had 20 nests positive with Calcitonin (Fig. 4F). This patient has been considered to be $\mathrm{C}$-cell hyperplasia because $\mathrm{C}$-cells in the normal thyroid gland is rather rare with about $0.1 \%$ of the total thyroid follicular cells population. C-cells belong to thyroid cysts and are located between the basal membrane and the follicular epithelium. Thus, the appearance of C-cells in the positions different from their normal site could be considered as C-cell hyperplasia [17].

Solid cell nests associated with HT may show some atypical nuclear features including prominent nuclear grooves, enlarged overlapping nuclei, and nuclear clearing. These features are sometimes mistaken for papillary thyroid microcarcinomas especially when the SCNs are numerous [9]. The nuclei of SCNs featured some worrisome aspects like chromatin clearing and nuclear membrane irregularity with occasional grooves and cup-shaped nuclei. These features may explain the misleading diagnosis of a papillary microcarcinoma performed by the first pathologist. The morphological details described previously were consistent with SCNs in a background of HT [19]. In the study by Bellevivine (2012), SCNs in a background of Hashimoto's thyroiditis was described as typical nuclear features of PTC, which became a trap for pathologists to distinguish them from micropapillary carcinoma on H.E staining slides. The author found that these foci were positive for p63 and confirmed these were SCNs and not micropapillary carcinoma [19]. In our study, $88.9 \%$ of SCNs had atypical nuclei mimicking PTC nuclear features. To further distinguish these SCNs from papillary microcarcinoma, p63 staining was performed and malignant characteristics were excluded like invasion and reactive stroma.

The immunohistochemical characteristics of SCNs were portrayed in detail by Reis-Filho in 2003. The most cells of solid cell nests showing a basal or stem cell phenotype are unequivocally brightened by p63, whereas C-cells and all other thyroid structures were consistently negative. Additionally, the most cells communicated carcinoembryonic antigen and all cytokeratins but for cytokeratin 20 and needed 
TTF-1, thyroglobulin, and Calcitonin. In differentiate, C-cells of solid cell nests showing features of parafollicular differentiation (positive for Calcitonin, AE1/AE3, and cytokeratin 7 and focal immunoreactivity for TTF-1 [20]. All cells of SCNs were positive for p63 and strongly positive for CK19 and TTF1 (Fig. 4C, E). Moreover, there was co-expression of CK19 and HBME-1 in the main cells of SCNs (94.4\%). According to Scognamiglio (2006), PTC can be distinguished from encapsulated follicular adenoma by papillary nuclear features, co-expression of CK19, and HBME-1, which has the specificity of $100 \%$ for the diagnosis of PTC [21]. As such immunophenotype of PTC expression, SCNs in Hashimoto's thyroiditis could be a malignant potential.

\section{Morphological and immunohistochemical characteristics of follicular cell changes}

Hashimoto's thyroiditis was moreover known as constant lymphocytic or autoimmune thyroiditis. The coexisting of HT with PTC has been taken note for decades. In any case, there's still no clear proof of whether HT was a chance that can advance the improvement of PTC. Histologically, in expansion to an eosinophilic variation of follicular cells which ordinarily related with thyroid follicular atrophy or damage, we seem moreover watch follicular cells dysplasia (FED) within the shape of scattered micro follicles missing follicular colloid or irregularly shaped follicles in HT tissues, particularly within the zones of thick encompassing lymphocytic infiltration. These follicles were as often as possible lined by Hürthle cells or cells with softly recolored cytoplasm. A few follicular cells were histologically comparative to PTC with extended, clear nuclear and grooves. Such injuries were ordinarily little without joining into a film. In a few cases, the expansion was more prominent, but still on the foundation of HT illnesses. In the study of 20 cases of HT, we found that follicular changes being seen in all cases surrounded focal lymphocytic thyroiditis or spread throughout the thyroid gland with the interstitial lymphoid infiltrates. The follicular cells having the PTC-like nuclear were seen in all 20 cases; Hürthle cell metaplasia, papillary, and glomerular-like structures accounted for $40 \%, 25 \%$, and $50 \%$ respectively (Table 3 ). Although, Hürthle cell metaplasia and PTC-like nuclear alterations with variant names like follicular epithelial dysplasia, clear nuclear changes in $\mathrm{HT}$ have been mentioned in many types of researches, papillary and glomerular- like structures were not noted in any studies (Fig. 3).

A study was conducted on 12 patients with $\mathrm{HT}$, who had clear nuclear changes like PTC of thyroid follicular cells. The monitoring of 12 patients in the period from 1.5-9 years showed that there was no evidence of recurrence as well as metastases. In this study, Arkadi (1995) declared that changes in thyroid follicular epithelium in HT, including PTC-like nuclear alterations, was the response of the thyroid follicular cells to the autoimmune process with potential malignancy. Thus, these changes should be examined context with the rest of the thyroid gland to avoid overdiagnosis of cancer [13].

In the research of Heng Ma (2014) performed on 18 patients with HT, there were scattered CK19 expression in follicular epithelial dysplasia, in particular, 5 cases $(27.8 \%)$ were moderate to strongly positive and 13 cases (72,7\%) were weakly positive. In Hürthle cells, the expression of CK19 protein was focal and weak; Moreover, most CK19- positive ones often located near lymphoma follicles and showing 
the extremely eosinophilic change. While there was only $11,1 \%$ of follicular epithelial dysplasia weakly positive to HBME-1 and negative to HBME-1 in Hürth cell metaplasia [4].

Another research performed on $23 \mathrm{HT}$ patients with focal or diffuse Hürthle cell change and PTC-like nuclear alterations of thyroid follicular cells showed that focal expression of cytokeratin 19 (CK19) and HBME- 1 was of $43 \%$ and $26 \%$, respectively and limited only in PTC-like nuclear alterations. There were four cases with PTC foci identified by strong and diffuse expression of markers specific to PTC, which promoted to retrospective studies examination of the hematoxylin and eosin-stained slides to fully evaluate atypical nuclear. Thus, such omitting lessons would be avoided. This study also showed that immunophenotypic changes of focal PTC-like nuclear alterations suggest the possibility of premalignant transformation in many cases of HT [22].

Hashimoto's thyroiditis suggests the possibility of early, focal premalignant transformation in some cases of Hashimoto's thyroiditis [21]. In our study, the thyroid follicular epithelial changes were negative for p63, Calcitonin, and positive for TTF1. These follicles with PTC-type nuclear changes were moderate to strong positive with CK19 (100\%), weak to moderate positive for HBME-1 (100\%) (Fig. 5A, B). Five cases (25\%), that had atypical solid nodule were strongly positive with CK19, moderately positive with HBME-1 (Fig. 5C, D), positive with TTF1 but negative with p63 and Calcitonin. Is it true that the follicular epitheliums or cell nests with PTC-like nuclear feature exhibited the molecular markers of PTC but did not show the molecular markers of SCNs are potential malignant transformations into PTC?

\section{Conclusions}

An increasing number of SCNs were detected in HT, mainly type 1 and type 4. SCNs located next to and in lymphoid follicles were mostly composed of main cells having PTC-like nuclear change, p63 and TTF1 positive expression, CK19 strongly and HBME-1 weakly positive staining.

In HT, there are many changes in follicular cells such as PTC-like nuclear alterations, Hürthle cell metaplasia, micropapillary and glomerular-like structure. All changes were negative with p63, Calcitonin and the majority of these changes were co-expressive with CK19 and HBME-1. Among these transformations, the micropapillary and glomerular-like structure had not been described in the previous literature. Moreover, another alteration being seen in HT was cell nests which are similar to SCNs in the morphology. They did not show p63 and Calcitonin but were strongly positive with CK19, moderately positive with HBME-1.

The co-expressed CK19 and HBME-1 of the main cells with PTC- like nuclear changes in SCNs and follicular epithelial changes, that may suggest precancerous lesions in Hashimoto's thyroiditis.

\section{Declarations}

\section{Authors contribution}


Tran Ngoc Dung; Nguyen Khac Tuyen: Conceptualization, Project Administration, Supervision, Validation.

Nguyen Thuy Linh: Conceptualization, Methodology, Writing - Review \& Editing; Corresponding; Last endorsement of the adaptation to be distributed

Do Nhu Binh, Nguyen Manh Hung: Data Curation, Formal Analysis, Investigation.

Truong Dinh Tien, Pham Van Thinh, Nguyen Khanh Van: interpretation of data, Methodology, writing Original Draft Preparation.

\section{Acknowledgments}

We thank all the staff in the Department of Histopathology, Military Medical Hospital 103 for collecting the samples.

\section{Ethical considerations}

The study was approved by the Ethical Review Committee of Vietnam Military Medical University. The study was in line with the Declaration of Helsinki. Written informed consent has been signed by all participants after full explanation.

\section{Disclosure}

The author(s) declared no potential conflicts of interest for the research, authorship, and/or publication of this article.

\section{Funding}

The author(s) received no financial support for the research, authorship, and/or publication of this article.

\section{References}

1. Caturegli P, De Remigis A, Chuang K, Dembele M, et al. Hashimoto's thyroiditis: celebrating the centennial through the lens of the Johns Hopkins hospital surgical pathology records. Thyroid. 2013;23 (2), 142-150.

2. LiVOLSI VA. The pathology of autoimmune thyroid disease: a review. Thyroid. 1994;4 (3), 333-339.

3. Paunovic I, Isic T, Havelka $M$, Tatic $S$, et al. Combined immunohistochemistry for thyroid peroxidase, galectin-3, CK19, and HBME-1 in differential diagnosis of thyroid tumors. Apmis. 2012;120 (5), 368379. 
4. Ma H, Yan J, Zhang C, Qin S, et al. Expression of papillary thyroid carcinoma-associated molecular markers and their significance in follicular epithelial dysplasia with papillary thyroid carcinoma-like nuclear alterations in Hashimoto's thyroiditis. Int J Clin Exp Pathol. 2014;7 (11), 7999-8007.

5. Segal K, Ben-Bassat M, Avraham A, Har-El G, et al. Hashimoto's thyroiditis and carcinoma of the thyroid gland. International surgery. 1985;70 (3), 205-209.

6. Eisenberg BL and Hensley SD. Thyroid cancer with coexistent Hashimoto's thyroiditis: clinical assessment and management. Archives of Surgery. 1989;124 (9), 1045-1047.

7. Ott RA, McCall AR, McHenry C, Jarosz $\mathrm{H}$, et al. The incidence of thyroid carcinoma in Hashimoto's thyroiditis. The American surgeon. 1987;53 (8), 442-445.

8. Ott RA, Calandra DB, McCall A, Shah KH, et al. The incidence of thyroid carcinoma in patients with Hashimoto's thyroiditis and solitary cold nodules. Surgery. 1985;98 (6), 1202-1206.

9. Sclafani AP, Valdes M and Cho H. Hashimoto's thyroiditis and carcinoma of the thyroid: optimal management. The Laryngoscope. 1993;103 (8), 845-849.

10. Okayasu I, Fujiwara M, Hara Y, Tanaka Y, et al. Association of chronic lymphocytic thyroiditis and thyroid papillary carcinoma. A study of surgical cases among Japanese, and white and African Americans. Cancer. 1995;76 (11), 2312-2318.

11. Burstein DE and et al. Immunohistochemical detection of p53 homolog p63 in solid cell nests, papillary thyroid carcinoma, and Hashimoto's thyroiditis: A stem cell hypothesis of papillary carcinoma oncogenesis. Hum Pathol. 2004. (36(5)), 590-1.

12. Hashimoto H. Zur kenntniss der lymphomatosen veranderung der Schilddruse (Struma lymphomatosa). Arch Klin Chir. 1912;97, 219-248.

13. Berho $M$ and Suster S. Clear nuclear changes in Hashimoto's thyroiditis. A clinicopathologic study of 12 cases. Ann Clin Lab Sci. 1995;25 (6), 513-21.

14. Asioli S, Erickson LA and Lloyd RV. Solid cell nests in Hashimoto's thyroiditis sharing features with papillary thyroid microcarcinoma. Endocrine pathology. 2009;20 (4), 197.

15. Manzoni M, Roversi G, Di Bella C, Pincelli Al, et al. Solid cell nests of the thyroid gland: morphological, immunohistochemical and genetic features. Histopathology. 2016;68 (6), 866-74.

16. Harach HR. Solid Cell Nests of the Human Thyroid in Early Stages of Postnatal Life. Cells Tissues Organs. 1986;127 (4), 262-264.

17. Autelitano F, Santeusanio G, Tondo UD, Costantino AM, et al. Immunohistochemical study of solid cell nests of the thyroid gland found from an autopsy study. Cancer. 1987;59 (3), 477-483.

18. Prod'hom $\mathbf{G}$ and Hedinger C (1985), Relationship between solid cell nests and focal lymphocytic thyroiditis, Annales de pathologie. 265-270.

19. Bellevicine C, Ippolito S, Arpaia D, Ciancia G, et al. Ultimobranchial Body Remnants (Solid Cell Nests) as a Pitfall in Thyroid Pathology. The Journal of Clinical Endocrinology \& Metabolism. 2012;97 (7), 2209-2210. 
20. Reis-Filho JS, Preto A, Soares P, Ricardo S, et al. p63 expression in solid cell nests of the thyroid: further evidence for a stem cell origin. Mod Pathol. 2003;16 (1), 43-8.

21. Scognamiglio T, Hyjek E, Kao J, and Chen YT. Diagnostic usefulness of HBME1, galectin-3, CK19, and CITED1 and evaluation of their expression in encapsulated lesions with questionable features of papillary thyroid carcinoma. American journal of clinical pathology. 2006;126 (5), 700-708.

22. Mizukami Y, Michigishi T, Nonomura A, Nakamura S, et al. Pathology of chronic thyroiditis: a new clinically relevant classification. Pathology annual. 1994;29, 135-158.

\section{Figures}

$\%$

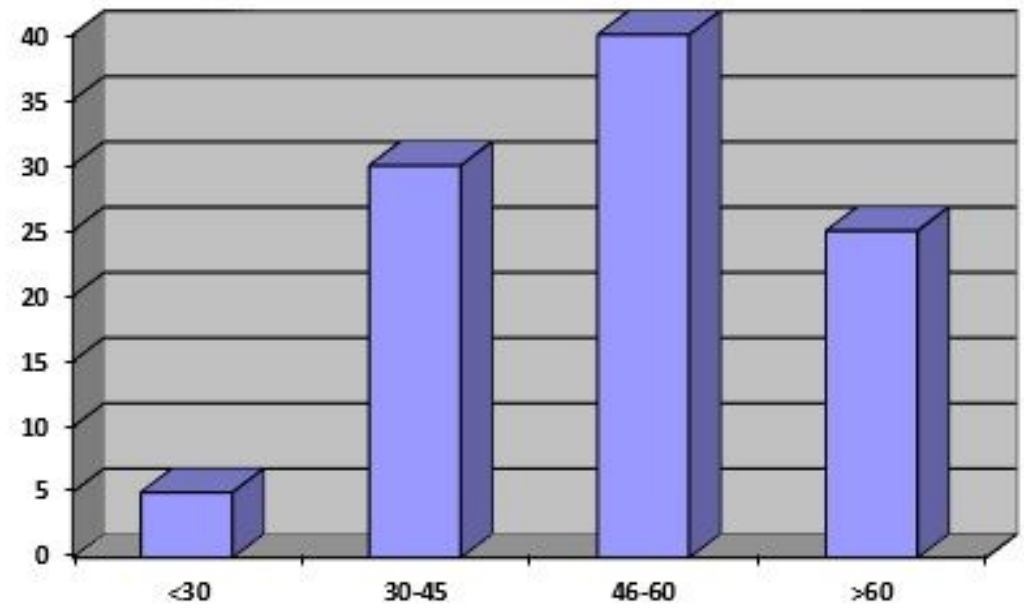

Figure 1

Age distribution of patients 

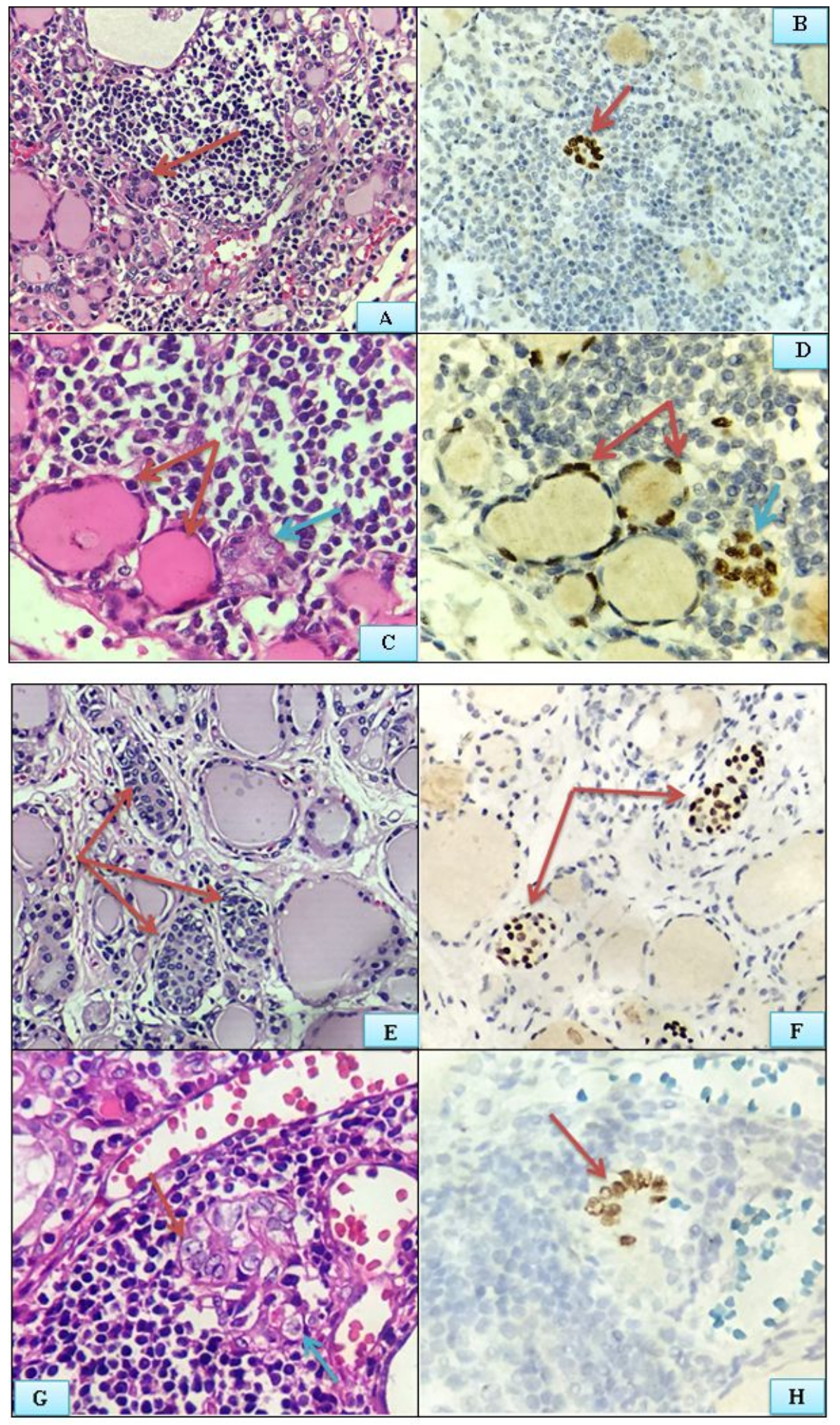

Figure 2

Morphological characteristics of solid cell nests (SCNs) A. Type 1 of SCNs HT: floret-like shape (Red arrow, Hematoxylin and Eosin (H\&E), Original magnification x 200) B. P63 positivity in type 1 of SCNs (Red arrow, Original magnification x 400) C. Type 4 of SCNs contain both solid of main cells (Blue arrow) and cystic of follicular epithelium (Red arrow), (H\&E, Original magnification x 400). D. P63 positivity in type 4 of SCNs (Red and blue arrow, Original magnification x 400) E. Type 1 of SCNs located between 
thyroid follicles (Red arrow, H\&E, Original magnification x 200) F. P63 positivity in type 1 of SCNs located between thyroid follicles (Red arrow, Original magnification x 400) G. PTC-like nuclear changes of SCNs: prominent nucleoli and nuclear groove (Red and blue arrow, H\&E, Original magnification x 400) H. P63 positivity in PTC-like nuclear changes of SCNs (Red arrow, Original magnification x 400)
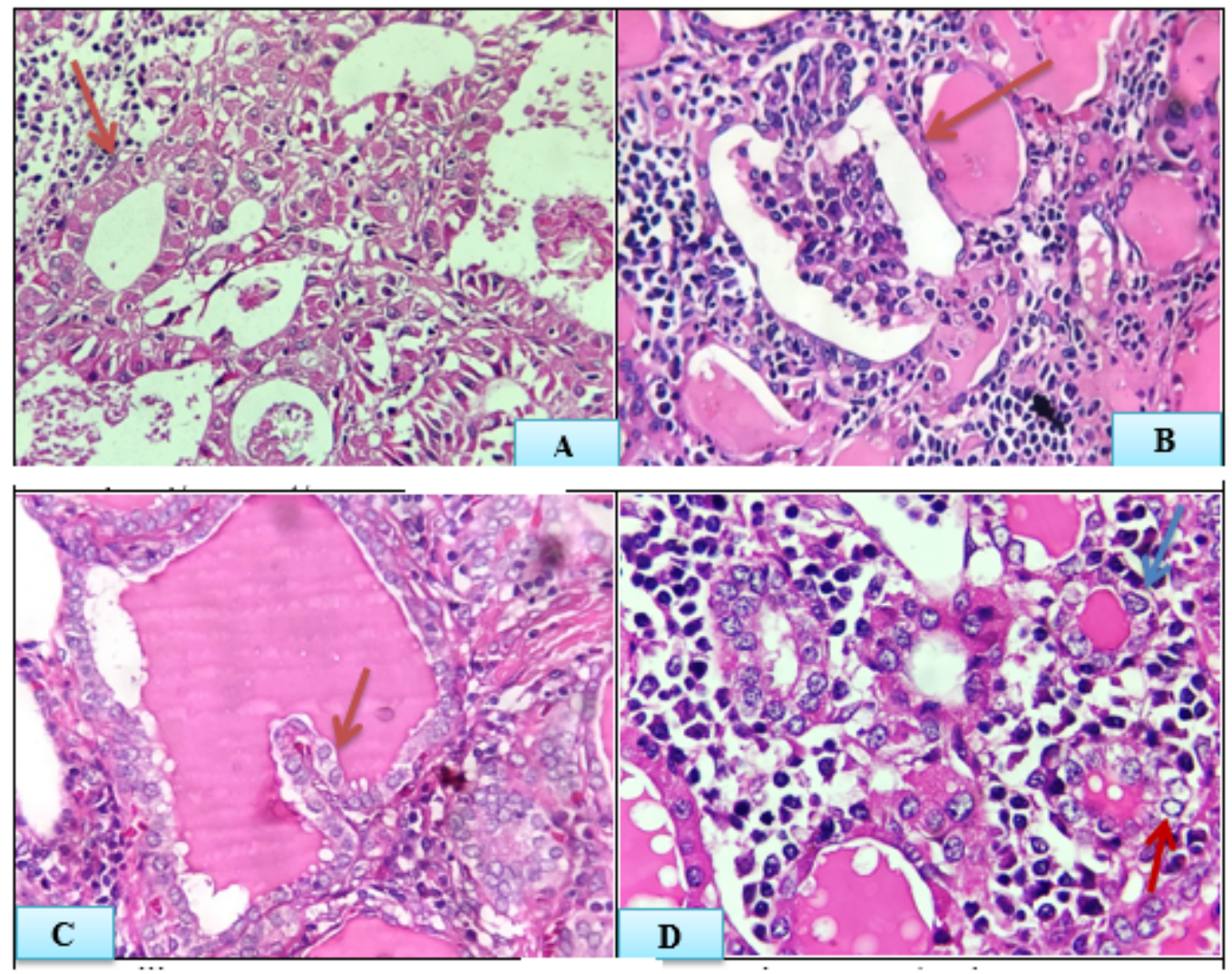

\section{Figure 3}

Histopathological characteristics of follicular cell changes A. Hürthle cell metaplasia in HT: Epithelial cells have eosinophilic, large cytoplasm (Red arrow), (H\&E, Original magnification x 200) B. Glomerularlike structure: grown from thyroid follicles which were damaged by lymphocytic infiltrations and inflammatory cells of interstitial tissues invade into these follicles forming the component in them similar to glomerulus (Red arrow, H\&E, Original magnification x 200) C. Papillary structure: Papillary with fibrovascular cores (Red arrow, H\&E, Original magnification x 400). D. PTC-like nuclear alterations: Nuclear groove (Blue arrow) and overlapping nuclear (Red arrow), (H\&E, Original magnification x 400) 


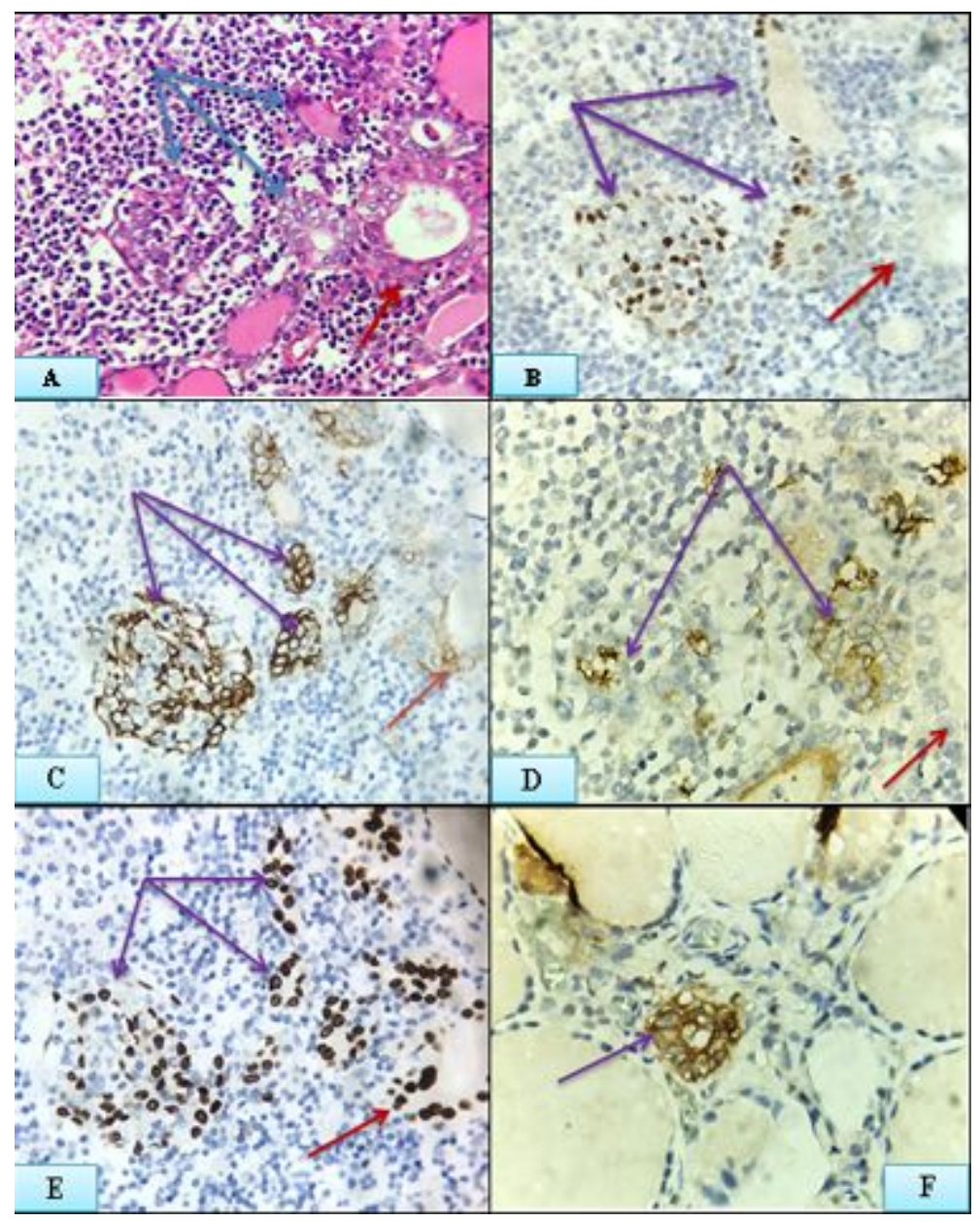

\section{Figure 4}

The immunohistochemical characteristics in SCNs A. SCNs (Blue arrow) and PTC-like nuclear alterations (Red arrow) are located next to the lymph follicles in HT (H\&E, Original magnification x 200) B. P63 positivity in SCNs (Purple arrow) and negativity in PTC-like nuclear alterations (Red arrow) (Original magnification x 200) C. CK19 positivity (3+) in SCNs (Purple arrow) and positivity (1+) in PTC-like nuclear alterations (Red arrow), (Immunoperoxidase, Original magnification x 200) D. HBME-1 positivity (2+) in SCNs (Purple arrow) and negativity in PTC-like nuclear alterations (Red arrow), (Immunoperoxidase, Original magnification x 200) E. TTF1 positivity in SCNs (Purple arrow) and PTC-like nuclear alterations (Red arrow), (Immunoperoxidase, Original magnification x 200) F. Calcitonin positivity in SCNs (Purple arrow, Immunoperoxidase, Original magnification x 400) 

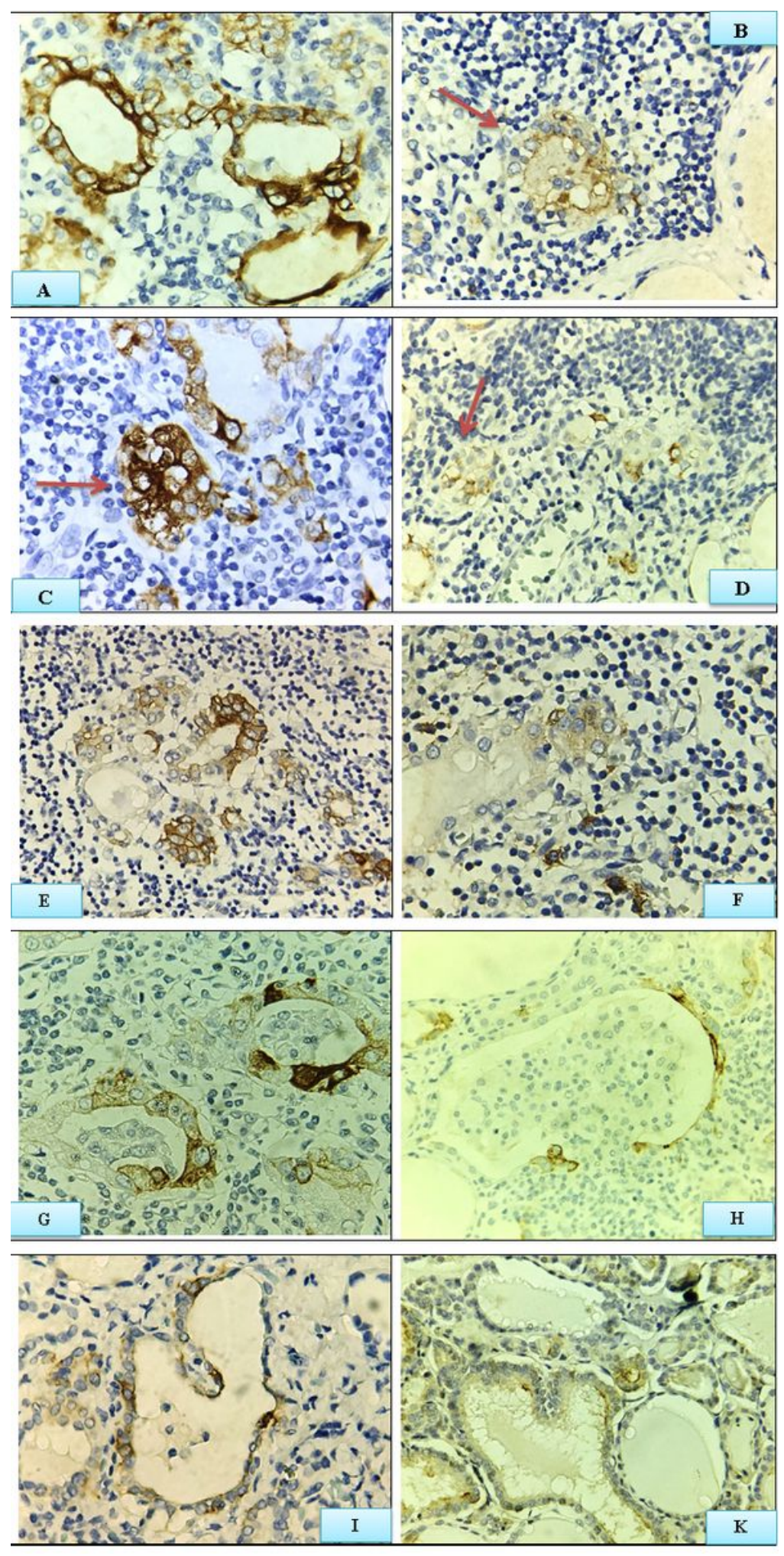

Figure 5

The immunohistochemical characteristics follicular cell changes A. CK19 positivity (3+) in Follicles formed PTC-like nuclear cells (Immunoperoxidase, Original magnification x 400) B. HBME-1 positivity (2+) in Follicles formed PTC-like nuclear cells (Red arrow, Immunoperoxidase, Original magnification x 400) C. CK19 positivity (3+) in an atypical solid nodule (Red arrow, Immunoperoxidase, Original magnification x 400) D. HBME-1 positivity (2+) in an atypical solid nodule (Red arrow, Immunoperoxidase, Original 
magnification $x$ 400) E. CK19 positivity (3+) in Hürthle cell metaplasia (Immunoperoxidase, Original magnification x 400 F. HBME-1 positivity (2+) in Hürthle cell metaplasia (Immunoperoxidase, Original magnification $x$ 400) G. CK19 positivity (3+) in the Glomerular-like structure (Immunoperoxidase, Original magnification $x 400 \mathrm{H}$. HBME-1 positivity (2+) in the Glomerular-like structure (Immunoperoxidase, Original magnification $x 400)$ I. CK19 positivity (2+) in papillary strucrue, (Immunoperoxidase, Original magnification $\times 400)$ K. HBME-1 positivity (2+) in papillary strucrue (Immunoperoxidase, Original magnification $\times 400$ ) 